\title{
INDEPENDENT RECURSIVE AXIOMATIZABILITY IN ARITHMETIC
}

\author{
J. P. JONES
}

1. Introduction. According to results of Tarski [7, p. 524] and Reznikoff [6], every theory may be axiomatized independently. However, there exist axiomatizable theories for which there are no recursively enumerable sets of independent axioms. The first example of such a theory was constructed by Kreisel [2].

Concerning formalized arithmetic, the well-known theories $R$ and $Q$ of [8] are easily seen to be independently recursively axiomatizable. Also, Peano arithmetic $P$ and each of its axiomatizable extensions is independently recursively axiomatizable. (This result follows from an old theorem of Mostowski [4], according to which the extensions of $P$ are reflexive theories. Montague and Tarski have shown [3] that axiomatizable reflexive theories are independently recursively axiomatizable.)

M. Poúr-El has proven [5] the existence of an axiomatizable nonindependently recursively axiomatizable extension of $Q$ which is compatible with $P$ (although not necessarily a subtheory of elementary arithmetic).

It is therefore natural to ask whether all axiomatizable subtheories of elementary arithmetic are independently recursively axiomatizable. By proving the following theorem we will show that this is not the case.

Theorem 1. There exist axiomatizable theories $T_{1}$ and $T_{2}$ such that

$$
R \subset T_{2} \subset Q \subset T_{1} \subset P
$$

and neither $T_{1}$ nor $T_{2}$ is independently recursively axiomatizable.

Kreisel's method [2] for constructing a nonindependently axiomatizable theory was to add a new predicate $P(x)$ to the syntax of $Q$ and the following axioms:

$$
(\forall x)[(\exists y) K(y, x) \rightarrow P(x)] \text { and } P\left(\Delta_{n}\right) \quad \text { for all } n
$$

where the formula $(\exists y) K(y, x)$ represents a hypersimple set in $Q$.

The idea of our construction is to replace the predicate $P(x)$ with a formula of arithmetic, $A(x)$. Roughly speaking, such a formula should have the property that its numeric substitution instances,

Received by the editors February 13, 1969. 
$A\left(\Delta_{0}\right), A\left(\Delta_{1}\right), \cdots$, are independent over some finitely axiomatizable theory in which the recursively enumerable sets may be represented.

Thus our construction is a refinement of that of Kreisel. We shall also make use of the following important result of Poúr-El [5]. Her Theorem sets forth the precise relationship between hypersimplicity and independent axiomatizability.

THEOREM 2. Let $\left\{\alpha_{0}, \alpha_{1}, \alpha_{2}, \cdots\right\}$ be any r.e. set of axioms for a theory $T$. Then $T$ is nonindependently recursively axiomatizable if and only if the set

$$
\left\{i:\left\{\alpha_{0}, \alpha_{1}, \cdots, \alpha_{i-1}\right\} \vdash \alpha_{i}\right\}
$$

is hypersimple.

2. Definitions and notation. All theories discussed in this paper, with the exception of Kreisel's, are formalized within a fixed first order predicate logic with identity, (specifically that of $[8$, p. 51]). This formalism has the following nonlogical constants: the individual constant symbol $\mathbf{0}$, the unary operation symbol $\boldsymbol{S}$ (successor), and the two binary operation symbols + and $\cdot$. As in [8], the formula $x \leqq y$ is an abbreviation for the formula $(\exists v)(v+x=y)$. In this formalism, the numerals, $\Delta_{n}$, are defined as follows: $\Delta_{0}=\mathbf{0}$ and $\Delta_{n+1}=\boldsymbol{S}\left(\Delta_{n}\right)$ for $0 \leqq n$.

For our purposes, a theory is simply any set of first order sentences on this formalism which contains the predicate calculus as a subset and is closed under logical deduction. Thus we identify a theory with the set consisting of its provable (valid) sentences. A theory is completely determined by specifying its (nonlogical) axioms. If $S$ and $T$ are theories with the same syntax and $S \subseteq T$ then we say that $S$ is a subtheory of $T$ and we write $S \subseteq T$. If $S \subseteq T$ and $S \neq T$ then we say $S$ is a proper subtheory of $T$ and we write $S \subset T$.

Let $\beta$ be a sentence and let $X$ be a set of sentences. If $\beta$ is provable in a theory $T$, we write $\vdash_{T} \beta$ or simply $\beta \in T$. If $\beta$ is deducible from the set $X$ within the theory $T$, we write $X \vdash_{T} \beta$. If $\beta$ is deducible from $X$ within the predicate calculus, we write $X \vdash \beta$. We usually omit brackets and simply present the elements of $X$ in a list.

Definition 1. A consistent set $X$ of sentences is said to be independent if for each $\beta \in X$

$$
X-\{\beta\} \not<\beta .
$$

DEFINITION 2. A theory $T$ is said to be independently recursively axiomatizable if there exists an independent recursive set of (nonlogical) axioms for $T$. 
REMARK. Craig's Theorem [1] asserts the equivalence of the notion of recursive axiomatizability with the notion of recursive enumerable axiomatizability. The notion of independent recursive axiomatizability and the notion of independent recursive enumerable axiomatizability are also equivalent. For if $\left\{\alpha_{1}, \alpha_{2}, \alpha_{3}, \ldots\right\}$ is any r.e. set of independent sentences, then the set consisting of the sentences $\alpha_{1}$, $\alpha_{2} \wedge \alpha_{2}, \alpha_{3} \wedge \alpha_{3} \wedge \alpha_{3}, \cdots$, is evidently independent and recursive (by virtue of increasing length).

Definition 3. A relation $R\left(x_{1}, x_{2}, \cdots, x_{k}\right)$ is said to be definable in a theory $T$ if there exists a formula $A\left(x_{1}, x_{2}, \cdots, x_{k}\right)$ with $k$ free variables such that

if $R\left(n_{1}, n_{2}, \cdots, n_{k}\right)$ then $\vdash_{T} A\left(\Delta_{n_{1}}, \Delta_{n_{2}}, \cdots, \Delta_{n_{k}}\right)$ and

if not $R\left(n_{1}, n_{2}, \cdots, n_{k}\right)$ then $\vdash_{T} \sim A\left(\Delta_{n_{1}}, \Delta_{n_{2}}, \cdots, \Delta_{n_{k}}\right)$.

Definition 4. A relation $R\left(x_{1}, x_{2}, \cdots, x_{k}\right)$ is said to be representable in a theory $T$ if there exists a formula $A\left(x_{1}, x_{2}, \cdots, x_{k}\right)$ with $k$ free variables such that

$$
R\left(n_{1}, n_{2}, \cdots, n_{k}\right) \text { if and only if } \vdash_{T} A\left(\Delta_{n_{1}}, \Delta_{n_{2}}, \cdots, \Delta_{n_{k}}\right) .
$$

Definition 5. A set $A$ of natural numbers is said to be hyperimmune if it is infinite and if no recursive function $f$ has the property that for each $n, f(n) \geqq$ the $n$th element of $A$ in increasing order. An r.e. set whose complement is hyperimmune is said to be hypersimple.

For reference we list the axioms for the three theories $R, Q$ and $P$ of [8]. We recall that $R \subset Q \subset P$.

Axioms of $R$ (SCHEMa).

1. $\Delta_{n}+\Delta_{m}=\Delta_{n+m}$,

2. $\Delta_{n} \cdot \Delta_{m}=\Delta_{n \cdot m}$,

3. $\Delta_{n} \neq \Delta_{m}$, if $n \neq m$,

4. $(\forall x)\left(x \leqq \Delta_{n} \rightarrow x=\Delta_{0} V_{x}=\Delta_{1} \bigvee \ldots \vee V x=\Delta_{n}\right)$,

5. $(\forall x)\left(x \leqq \Delta_{n} \vee \Delta_{n} \leqq x\right)$.

Axioms of $Q$.

1. $(\forall x)(\forall y)(S(x)=S(y) \rightarrow x=y)$.

2. $(\forall y)(S(y) \neq 0)$,

3. $(\forall x)(x \neq 0 \rightarrow(\exists y)(x=S(y)))$,

4. $(\forall x)(x+0=x)$,

5. $(\forall x)(\forall y)(x+S(y)=S(x+y))$,

6. $(\forall x)(x \cdot 0=0)$,

7. $(\forall x)(\forall y)(x \cdot S(y)=x \cdot y+x)$.

Axioms of $P$ (Peano's arithmetic).

1. The seven axioms of $Q$, and 
2. all sentences which are particular instances of the following induction scheme:

$$
\Phi(O) \wedge(\forall u)[\Phi(u) \rightarrow \Phi(S(u))] \rightarrow(\forall u) \Phi(u) .
$$

3. Preliminaries. Let $H$ be a fixed hypersimple set of natural numbers which, for convenience, does not contain 0 . Let $L(y, x)$ be a recursive binary relation with the property that, for every natural number $n$,

$n \in H$ if and only if there exists an $m$ such that $L(m, n)$.

( $L$ could be taken to be the graph of a recursive function which enumerates $H$.) According to $[8$, p. 56], every recursive set is definable in $R$. Thus we may choose a fixed formula $K(y, x)$ with two free variables which defines the relation $L(y, x)$ in $R$. The formula $K(y, x)$ will be used in the axiomatization of $T_{1}$ and $T_{2}$.

4. Construction of $T_{1}$. Let $A_{1}(x)$ and $F_{1}(x)$ be abbreviations for the following two formulas:

$$
\Lambda_{1}(x)=(\forall z)(S(z)=z \rightarrow x \cdot z=z), \quad F_{1}(x)=S(x) \neq x .
$$

$A_{1}(x)$ will play the role played by $P(x)$ in Kreisel's construction.

Axioms of $T_{1}$.

$\sigma_{1}$. The conjunction of the seven axioms of $Q$,

$\Sigma_{1} .(\forall x)\left[F_{1}(x) \wedge(\exists y) F_{1}(y) \wedge K(y, x) \rightarrow A_{1}(x)\right]$, $A_{1}\left(\Delta_{n}\right)$ for all $n$.

Now the sentence $(\forall x) A_{1}(x)$ is evidently provable in $P$ (by induction). Consequently we have $Q \subseteq T_{1} \subseteq P$.

The following lemma establishes the independence of the sentences $A_{1}\left(\Delta_{n}\right)$ in a quite strong sense. Here we make heavy use of the fact that $Q$ has nonstandard models.

Lemma 1. For each set $M$ of natural numbers there exists "model $\dddot{M}_{1}(M)$ of $Q$ with the following properties:

(i) The sentence $A_{1}\left(\Delta_{m}\right)$ is true in the model if and only if $m \in .1 \%$.

(ii) The sentence $\Sigma_{1}$ is true in the model if and only if $H \subseteq M$.

Proor. The domain of the model is to be the set

$$
\left\{0,1,2, \cdots, \infty_{0}, x_{1}\right\} \text {. }
$$

The operations $S,+$, and are defined in the usual way upon the natural numbers. We define $n+\infty_{i}=\infty_{i}+n=\infty_{i}, \infty_{i}+\infty_{j}=\infty_{i} \cdot \infty_{j}$ $=\infty_{i}$, and $S\left(\infty_{i}\right)=\infty_{i}$. Also, for a natural number $n$, 


$$
\begin{aligned}
& \infty_{i} \cdot n=\infty_{i} \quad \text { if } 0<n, \quad n \cdot \infty_{i}=\infty_{i} \quad \text { if } n \in M \text {, } \\
& =0 \quad \text { if } 0=n ; \quad=\infty_{1-i} \quad \text { if } n \notin M \text {. }
\end{aligned}
$$

It is routine to check that $\mathfrak{M}_{1}(M)$, so defined, is in fact a model of $Q$ and that property (i) obtains. To see that property (ii) holds, observe that the interpretation of $F_{1}(y)$ in this model is that " $y$ is a natural number." Now the formula $K(y, x)$ defines the relation $L(y, x)$ in the theory $Q$ and $\mathfrak{T M}_{1}(M)$ is a model of $Q$. Therefore $(\exists y) F_{1}(y) \wedge K\left(y, \Delta_{m}\right)$ is true in the model if and only if $m \in H$. So by property (i), $\Sigma_{1}$ is true in the model if and only if $H \subseteq M$. This completes the proof of Lemma 1.

We show that $T_{1}$ is nonindependently recursively axiomatizable. Consider the following axiomatization $\alpha_{n}$ of $T_{1}$ :

$$
\alpha_{0}=\sigma_{1} \wedge \Sigma_{1} \wedge A\left(\Delta_{0}\right), \quad \alpha_{n}=A\left(\Delta_{n}\right) \text { for } n=1,2,3, \cdots .
$$

We use Theorem 2 of $\S 1$. It suffices to prove that $H=I$ where $H$ is the hypersimple set of $\S 3$ and $I=\left\{i:\left\{\alpha_{0}, \alpha_{1}, \cdots, \alpha_{i-1}\right\} \vdash \alpha_{i}\right\}$.

From the way in which the $\alpha_{n}$ was defined, we have that $i \in I$ if and only if $0<i$ and

(iii) $\Sigma_{1}, A_{1}\left(\Delta_{0}\right), A_{1}\left(\Delta_{1}\right), \cdots, A_{1}\left(\Delta_{i-1}\right) \vdash_{Q} A_{1}\left(\Delta_{i}\right)$.

To see that $H \subseteq I$, suppose $i \in H$. Then $0<i$ and there exists an $n$ such that $L(n, i)$ and hence $\vdash_{Q} K\left(\Delta_{n}, \Delta_{i}\right)$. Consequently $\Sigma_{1} \vdash_{Q} A_{1}\left(\Delta_{i}\right)$ and so $i \in I$.

To see that $I \subseteq H$, suppose $i \in I$. Then $0<i$ and (iii) holds. Let $M=H \cup\{0,1, \cdots, i-1\}$ and consider the model $\mathfrak{M}_{1}(M)$ of Lemma 1. By properties (i) and (ii) of Lemma 1 the antecedents, $\Sigma_{1}, A\left(\Delta_{0}\right), A\left(\Delta_{1}\right), \cdots, A\left(\Delta_{i-1}\right)$, are true in this model. Consequently, $A\left(\Delta_{i}\right)$ is true in the model because, according to (iii), it is deducible from sentences true in the model. So by property (i) of Lemma 1 we have $i \in M$. So $i \in H$ and we have shown that $I=H$.

5. Construction of $T_{2}$. Let $A_{2}(x)$ and $F_{2}(x)$ be abbreviations for the following two formulas:

$$
A_{2}(x)=(\forall z)(x \cdot S(z)=x \cdot z+x), \quad F_{2}(x)=(x=0 \vee(\exists u)(x=S(u))) .
$$

We wish to construct a nonindependently axiomatizable theory $T_{2}$ such that $R \subset T_{2} \subset Q$.

However, the theory $R$ is not finitely axiomatizable $[8$, p. 55] and this particular property of $Q$ was crucial in our construction of $T_{1}$. For this reason, we construct first a finitely axiomatizable extension $Q^{\prime}$ of $R$ such that $R \subset Q^{\prime} \subset Q$. We then construct $T_{2}$ so that $R \subset Q^{\prime}$ $\subset T_{2} \subset Q$. 
Axioms of $Q^{\prime}$.

1. $(\forall x)(\forall y)\left(F_{2}(x) \wedge F_{2}(y) \rightarrow(\boldsymbol{S}(x)=\mathbf{S}(y) \rightarrow x=y)\right)$,

2. $(\forall x)(S(x) \neq 0)$,

3. $(\forall x)(x+0=x)$,

4. $(\forall x)(\forall y)\left(F_{2}(x) \wedge F_{2}(y) \rightarrow(x+S(y)=S(x+y))\right)$,

5. $(\forall x)\left(F_{2}(x) \rightarrow x \cdot 0=0\right)$,

6. $(\forall x)(\forall y)\left(F_{2}(x) \wedge F_{2}(y) \rightarrow(x \cdot S(y)=x \cdot y+x)\right)$,

7. $(\forall x)(\forall y)\left(\sim F_{2}(x) \rightarrow y \leqq x\right)$,

8. $(\forall x)(\forall y)\left(F_{2}(x) \wedge F_{2}(y) \leftrightarrow F_{2}(x+y)\right)$,

9. $(\forall x)\left(\left(F_{2}(x) \wedge x \neq 0\right) \rightarrow(\exists z)\left(F_{2}(z) \wedge x=S(z)\right)\right)$.

Axioms of $T_{2}$.

$\sigma_{2}$. The conjunction of the nine axioms of $Q^{\prime}$,

$\Sigma_{2} .(\forall x)\left[F_{2}(x) \wedge(\exists y) F_{2}(y) \wedge K(y, x) \rightarrow A_{2}(x)\right]$, $A_{2}\left(\Delta_{n}\right)$ for all $n$.

We claim that $R \subseteq Q^{\prime} \subseteq T_{2} \subseteq Q$. Evidently $Q^{\prime} \subseteq T_{2} . T_{2} \subseteq Q$ because the sentences $(\forall x)\left(F_{2}(x)\right)$ and $(\forall x) A_{2}(x)$ are axioms of $Q$. To check that $R \subseteq Q^{\prime}$ is straightforward but tedious. We omit the details. As before we make use of a nonstandard model.

Lemma 2. For each set $M$ of natural numbers there exists a model, $\mathfrak{M}_{2}(M)$ of $Q^{\prime}$ with the following properties:

(i) The sentence $A_{2}\left(\Delta_{m}\right)$ is true in the model if and only if $m \in M$.

(ii) The sentence $\Sigma_{2}$ is true in the model if and only if $H \subseteq M$.

Proof. The domain of the model is to be the set $\{0,1,2, \cdots, \infty\}$. The operations $S,+$ and $\cdot$ are defined in the usual way upon the natural numbers. We define $\infty+\infty=\infty, \infty \cdot \infty=n+\infty=\infty+n=\infty$. However, $S(\infty)=2$. We multiply as follows:

$$
\begin{array}{rlrl}
\infty \cdot n=n \cdot \infty & =n & & \text { if } n \in M, \\
& =n+1 & \text { if } n \in M .
\end{array}
$$

It is easy to check that $\mathfrak{T}_{2}(M)$ is a model of $Q^{\prime}$ and that property (i) holds. As for property (ii), the argument given in the proof of Lemma 1 . $\$ 4$ will establish this, if, in this argument, we replace $Q$ by $Q^{\prime}$ and change subscripts from 1 to 2 . This completes the proof of Lemma 2.

Similarly, the argument to the effect that $T_{2}$ is nonindependently recursively axiomatizable is identical with the argument given in $\$ 4$ for $T_{1}$. This completes the construction of $T_{2}$. 


\section{REFERENCES}

1. W. Craig, On axiomatizability within a system, J. Symbolic Logic 18 (1953), 30-32. 109.

2. G. Kreisel, Independent recursive axiomatization, J. Symbolic Logic 22 (1957),

3. R. Montague and A. Tarski, Independent recursive axiomatizability, Mimeographed notes, Summer Institute for Symbolic Logic, Cornell University, Ithaca, N. Y., 1957, p. 270.

4. A. Mostowski, On models of axiomatic systems, Fund. Math. 39 (1952), 133-158.

5. M. B. Pour-El, Hypersimplicity as a necessary and sufficient condition for nonindependent axiomatization, Z. Math. Logik Grundlagen Math. 14 (1968), 449-456.

6. I. Reznikoff, Tout ensemble de formules de la logique classique est équivalent a un ensemble independent, C. R. Acad. Sci. Paris 260 (1965), 2385-2388.

7. A. Tarski, Grundzüge des systemenkalkuls. I, Fund. Math. 25 (1935), 503-526.

8. A. Tarski, A. Mostowski and R. Robinson, Undecidable theories, North-Holland, Amsterdam, 1953, ix +98 pp.

UNIVERSITY OF CALGaRy 